\title{
BUCKLING OF CIRCULAR PLATES WITH SHELL-STIFFENING ON THE BOUNDARY
}

\author{
DÁNIEL BURMEISTER \\ Institute of Applied Mechanics, University of Miskolc \\ H-3515 Miskolc, Miskolc-Egyetemváros, Hungary \\ daniel. burmeister@uni-miskolc.hu
}

[Received: January 26, 2015, Accepted: April 15, 2015]

\begin{abstract}
The present paper is concerned with the stability problems of a thin solid circular plate and some annular plates, each stiffened by a cylindrical shell on the external boundary. Assuming an axisymmetric dead load and non-axisymmetric deformations we determine the critical load in order to clarify what effect the stiffening shell has on the critical load. Using Kirchhoff's theory of thin shells and plates the paper presents the governing equations both for the circular plate and for the cylindrical shell, where the displacement field of the shell is obtained from a Galerkin function. The deflection of the plate and the Galerkin function are expanded into Fourier series and consequently all physical quantities in the structural elements as well. The boundary- and continuity conditions and last but not least numerical results are also presented.
\end{abstract}

Mathematical Subject Classification: 74K20, 65L15

Keywords: Buckling, stability problem, shell-stiffened circular plates, cylindrical shell, Galerkin function

\section{INTRODUCTION}

Stability investigations of engineering structures look back on a long history. As regards the stability problem of circular plates, we mention that the first paper devoted to this issue was published in 1890 [1. Since then a number of papers have been devoted to this problem. Without aiming at completeness here we cite some important papers 211 .

There are various methods for increasing the resistance of a circular plate to buckling. For example, one can apply an internal ring support, which can be either rigid or elastic. Thevendran and Wang have examined the buckling problem of annular plates which are simply supported with elastic rotational restraints at the inner or outer boundary [12. Laura et al. have investigated the buckling of circular, solid and annular plates with an intermediate circular support under the assumption of axisymmetric deformations [13. By the use of the Kirchhoff-Love plate theory [14,15] and the Mindlin-Reissner theory 16] Wang and his co-authors studied the same structure under the assumption of non-axisymmetric deformations. Rao and Rao have analysed 
the buckling of circular plates which are supported along concentric rings. The supports applied are simple or translational and/or torsional elastic restraints $17-20$. The authors have also investigated a circular plate with elastic foundation [21].

Another method to increase stability of a circular plate is the use of stiffeners. If these are applied densely, their effect can be averaged and smeared out by using orthotropic plates. The theoretical background of this technique can be found in the book written by Troitsky 22. Buckling of such stiffened plates has been examined among others by Simitses and Blackmon 23] and Srinivasan and Thiruvenkatachari [24.

We can also use discrete stiffeners. The effect on stability of a ring stiffener on the boundary of a circular plate is investigated by Phillips and Carney 25. A ring stiffened circular plate is investigated in a paper by Turvey and Der Avanessien [26]. The paper cited is concerned among others with experimental results. However, the stability issues are left out of consideration. A further paper by Turvey and Salehi [27] deals with an annular plate stiffened by a single diameter stiffener. The stability problem is, however, again left out of consideration. A further paper by Golmakani and Mehrabian deals with deflection analysis of axisymmetric ring-stiffened circular and annular laminated plates subjected to a transverse uniform load 28].

Rossettos and Miller have investigated symmetric and asymmetric buckling of a circular plate which is stiffened by a ring at an internal radius 29,30. The axial rigidity of the stiffening ring has been ignored. Frostig and Simitses have examined a similar structure but they have not used the simplifications of the aforementioned article 31,32$]$. The stiffening ring is modeled as a curved beam.

Szilassy dealt with the stability of circular and annular plates stiffened by a cylindrical shell on its outer boundary in his PhD. thesis [33] and in a further article [34]. It was assumed that (i) the load is an in-plane axisymmetric dead one and (ii) the deformations of the annular plate and the cylindrical shell are also axisymmetric. For solving the corresponding eigenvalue problem, he used the solution of a differential equation set up for the rotation field while the solution for the cylindrical shell is based on the theory of thin shells.

The present paper deals with the non-axisymmetric buckling of circular and annular plates which are stiffened by a cylindrical shell on the outer boundary. The paper outlines the basic assumptions, the governing equations as well as the boundary and continuity conditions. Numerical results are also shown. These represent the influence of shell geometry on buckling load. As regards the cylindrical shell we shall utilize some results from Vlasov 35 and Jezsó 36].

\section{Problem formulation}

The cross section of the shell-stiffened structure we are concerned with is shown in Figure 1. It consists of either a solid circular plate or an annular one - the latter is shown in Figure 1 - and a cylindrical shell, which stiffens the plate on its external boundary. The inner radius of the plate is denoted by $R_{i}$, the radius of the intersection 
line of the middle surfaces of the plate and the shell by $R_{e}$. If the plate is a circular one then $R_{i}=0$. The shell is symmetric with respect to the middle plane of the plate. Its height is $2 h$. We shall assume that $R_{e}$ coincides with the external radius of the plate. The thicknesses of the plate and shell are denoted by $b_{p}$ and $b_{s}$, respectively. The structure is loaded by radial distributed forces with a constant intensity $f_{o}$ acting in the middle plane of the plate. The load is a dead load.

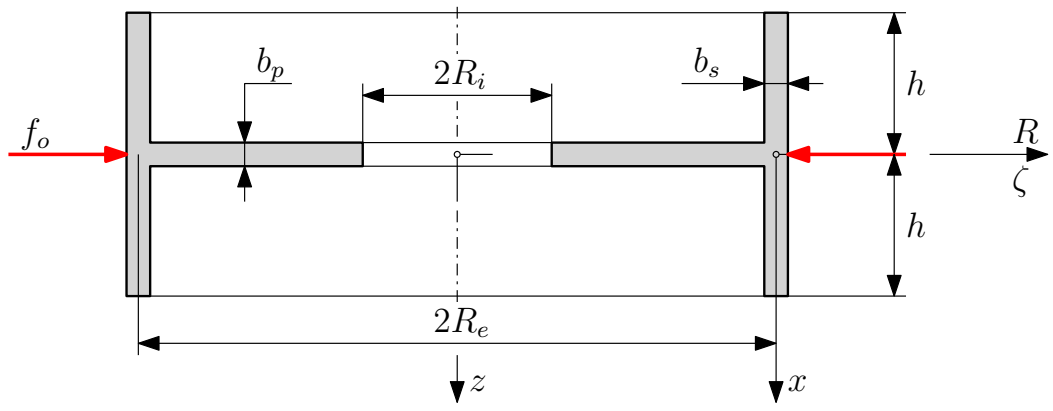

Figure 1. The structure and its load

We shall assume that the plate and the shell are thin, consequently we can use the Kirchhoff theory of plates and shells. It is also assumed that the problem is linear with regard to the kinematic equations and material law. Heat effects are not taken into account. The plate and the shell are made of homogeneous isotropic material for which $E_{p}, E_{s}$ and $\nu_{p}, \nu_{s}$ are Young's modulus and Poisson's ratio for the plate and the shell respectively.

Figure 2 shows the various tasks which can be investigated. Figure $2 a$. is the inner part of a solid circular plate and subfigures b.-d. are supports that can be applied on the inner boundary of an annular plate.

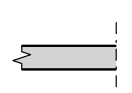

a.

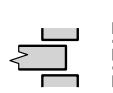

b.

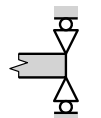

c.

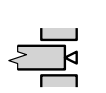

d.

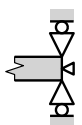

e.

Figure 2. Possible supports

Our main goal is to determine (a) the critical load of the structure and (b) the effect of the stiffening shell on the critical load under the assumption of small, nonaxisymmetric and linearly elastic deformations.

The shell and the plate are divided mentally. The cylindrical coordinate system $(R, \varphi, z)$ is used for the equations of the plate - the plane $z=0$ coincides with the middle surface of the plate. Figure 3 a. shows the corresponding coordinate curves on the circle with radius $R_{e}$. The displacements on the middle surface in the directions $R, \varphi$ and $z$ are denoted by $u, v$ and $w$ respectively. 
For the cylindrical shell the coordinate system $(\zeta, \varphi, \xi)$ is applied. The coordinate surface $\xi=z, \zeta=0$ coincides with the middle surface of the shell with radius $R_{e}$. The polar angle $\varphi$ is the same in the two coordinate systems. The coordinate curves on the middle surface of the shell are also shown in Figure $3 \mathrm{~b}$. The displacements on the middle surface of the shell in the directions $\zeta, \varphi, \xi$ are denoted by $u_{\zeta}, u_{\varphi}$ and $u_{\xi}$, respectively.

In the mathematical formulations we use the dimensionless coordinates $\rho=R / R_{e}$ and $\xi=x / R_{e}$. The dimensionless coordinates on the boundaries are denoted by $\rho_{i}=R_{i} / R_{e}$ and $\xi_{h}=h / R_{e}$.

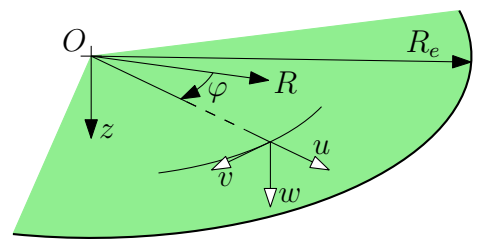

a.

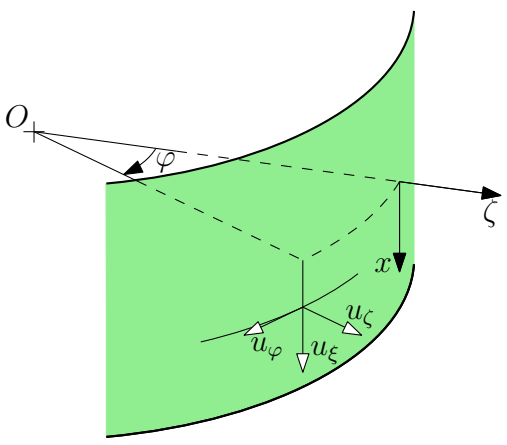

b.

Figure 3. Coordinate curves in the coordinate systems

\section{Governing EQUATions}

3.1. In-plane loads and forces. Figure 4 shows the separated parts of the structure with the inner forces acting in the middle plane of the plate between the two elements. The load is axisymmetric, consequently the equations governing the prebuckling (membrane) state for each part are axisymmetric as well.

a.

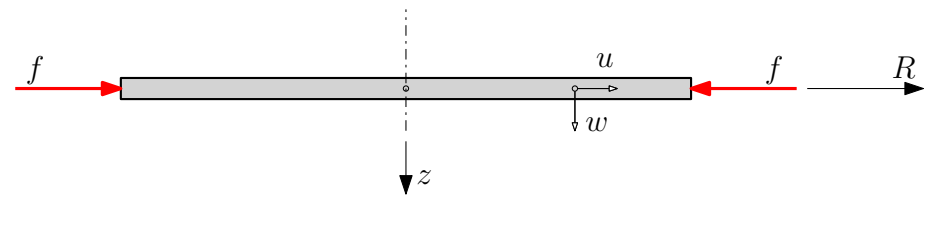

b.

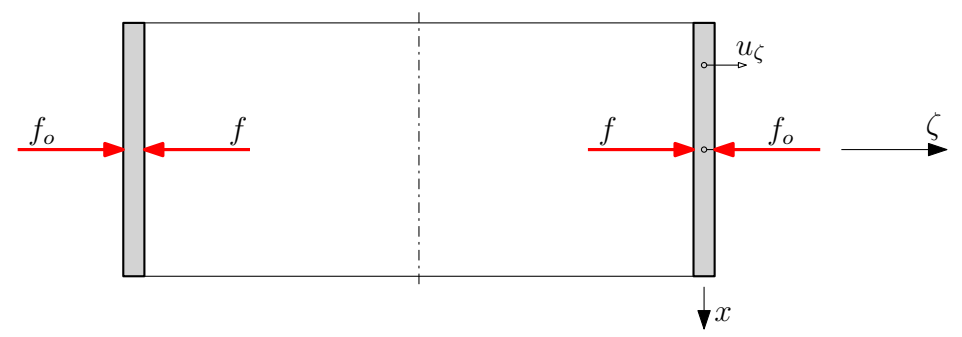

Figure 4. Free body diagram for plate and shell 
The distributed load $f$ exerted on the plate by the shell can be obtained from the axisymmetric deformations of the shell and by utilizing the continuity condition between the shell and the plate:

$u(\rho=1)=u_{\zeta}(\xi=0)=-\underbrace{\frac{\nu_{o}}{2 E_{s}}\left(\frac{R_{e}}{b_{s}}\right)^{\frac{3}{2}} \frac{\cos 2 h \beta+\cosh 2 h \beta+2}{\sin 2 h \beta+\sinh 2 h \beta}}_{\alpha}\left(f_{o}-f\right)=-\alpha\left(f_{o}-f\right)$.

where $\nu_{o}=\sqrt[4]{3\left(1-\nu_{s}^{2}\right)}$ and $\alpha$ is defined by the above relation - for details see 37.

Due to the axisymmety of the in-plane loads, the inner forces in the plate depend only on the radial coordinate $\rho$. The inner forces due to $f$ take the form

$$
\begin{aligned}
N_{R} & =f \mathcal{N}_{R}=f\left(-A+\frac{B}{\rho^{2}}\right), \\
N_{\varphi} & =f \mathcal{N}_{\varphi}=f\left(-A-\frac{B}{\rho^{2}}\right), \\
N_{R \varphi} & =N_{\varphi R}=f \mathcal{N}_{R \varphi}=0
\end{aligned}
$$

where the constants $A$ and $B$ depend on the boundary conditions. It follows from the axisymmetry that $N_{R \varphi}$ vanishes. If the plate is a solid one - see Figure $2 \mathrm{a}$ - then

$$
A=1 \quad \text { and } \quad B=0 .
$$

If the inner boundary is free and $f$ is the distributed load on the outer boundary see Figure 2b. and c. - then the constants are as follows:

$$
A=\frac{1}{1-\rho_{i}^{2}} \quad \text { and } \quad B=\frac{\rho_{i}^{2}}{1-\rho_{i}^{2}} .
$$

If the radial displacement vanishes on the inner boundary - see Fig. 2 d. and e. then

$$
A=\frac{1+\nu_{p}}{1+\nu_{p}+\rho_{i}^{2}\left(1-\nu_{p}\right)} \quad \text { and } \quad B=-\frac{\rho_{i}^{2}\left(1-\nu_{p}\right)}{1+\nu_{p}+\rho_{i}^{2}\left(1-\nu_{p}\right)} .
$$

The radial displacement on the inner boundary can be calculated by using the relations

$$
u(\rho=1)=-K \frac{R_{e}}{b_{p}} \frac{f}{E_{p}}
$$

where the constant $K$ depends on the boundary conditions

$$
\begin{array}{rlrl}
K & =1-\nu_{p} & \text { if } & \rho_{i}=0, \\
K=\frac{1-\nu_{p}+\rho_{i}^{2}\left(1+\nu_{p}\right)}{1-\rho_{i}^{2}} & \text { if } & N_{R}\left(\rho=\rho_{i}\right)=0, \\
K=\frac{\left(1-\nu_{p}^{2}\right)\left(1-\rho_{i}^{2}\right)}{1+\nu_{p}+\rho_{i}^{2}\left(1-\nu_{p}\right)} & \text { if } & u\left(\rho=\rho_{i}\right)=0 .
\end{array}
$$


3.2. Deformation of the annular plate, equations for the displacement field after stability loss. The buckling equation can be given in the form 32

$$
\Delta \Delta w-\mathfrak{F}\left[\mathcal{N}_{R} \frac{\partial^{2} w}{\partial \rho^{2}}+\mathcal{N}_{\varphi}\left(\frac{1}{\rho} \frac{\partial w}{\partial \rho}+\frac{1}{\rho^{2}} \frac{\partial^{2} w}{\partial \varphi^{2}}\right)\right]=0
$$

where

$$
\mathfrak{F}=f \frac{R_{e}^{2}}{I_{1 p} E_{1 p}}
$$

is the dimensionless load parameter wherein

$$
I_{1 p}=\frac{b_{p}^{3}}{12}, \quad E_{1 p}=\frac{E_{p}}{1-\nu_{p}^{2}}
$$

and

$$
\Delta=\frac{\partial^{2}}{\partial \rho^{2}}+\frac{1}{\rho} \frac{\partial}{\partial \rho}+\frac{1}{\rho^{2}} \frac{\partial^{2}}{\partial \varphi^{2}}
$$

is the Laplace operator in the polar coordinate system $(\rho, \varphi)$.

The physical quantities which appear in the boundary conditions, such as the rotation, the shear force and the bending moment can all be given in terms of the solution for $w$ as follows

$$
\begin{gathered}
\vartheta=-\frac{1}{R_{e}} \frac{\mathrm{d} w}{\mathrm{~d} \rho}, \\
M_{R}=-\frac{I_{1 p} E_{1 p}}{R_{e}^{2}}\left[\frac{\partial^{2} w}{\partial \rho^{2}}+\frac{\nu_{p}}{\rho}\left(\frac{\partial w}{\partial \rho}+\frac{1}{\rho} \frac{\partial^{2} w}{\partial \varphi^{2}}\right)\right], \\
Q_{R}=I_{1 p} E_{1 p} \frac{1}{R_{e}^{3}} \frac{\partial}{\partial \rho}(\Delta w)-\frac{N_{R}}{R_{e}} \frac{\partial w}{\partial \rho}-\frac{1}{\rho R_{e}} \frac{\partial M_{R \varphi}}{\partial \varphi} .
\end{gathered}
$$

For non axisymmetric deformations we expand the solution giving the deflection $w$ in a Fourier series of the form

$$
w=w_{o}+\sum_{m=0}^{1} \sum_{n=1}^{\infty} \stackrel{m}{w}_{n}(\rho) \cos \left(n \varphi-m \frac{\pi}{2}\right)
$$

and substitute it into 3.9$)$. We obtain that the amplitudes $w_{o}(\rho)$ and $\stackrel{m}{w}_{n}(\rho)$ should fulfill the following differential equations:

$$
\begin{gathered}
\Delta_{n} \Delta_{n} \stackrel{m}{w}_{n}-\mathfrak{F}\left[\mathcal{N}_{R} \frac{\partial^{2}{ }^{m} w_{n}}{\partial \rho^{2}}+\mathcal{N}_{\varphi}\left(\frac{1}{\rho} \frac{\partial w_{n}}{\partial \rho}-\frac{n^{2}}{\rho^{2}} w_{n}\right)\right]=0 \\
m=0,1 ; \quad n=1,2, \ldots
\end{gathered}
$$

where

$$
\Delta_{n}=\frac{\mathrm{d}^{2}}{\mathrm{~d} \rho^{2}}+\frac{\mathrm{d}}{\rho \mathrm{d} \rho}-\frac{n^{2}}{\rho^{2}} .
$$

If the plate is a circular one, than $\mathcal{N}_{R}=\mathcal{N}_{\varphi}=1$, and equations 3.12 have a closed form solution:

$$
\stackrel{m}{w}_{n}(\rho)=c_{1} Z_{1}+c_{2} Z_{2}+c_{3} Z_{3}+c_{4} Z_{4}
$$




$$
Z_{1}=\rho^{n}, \quad Z_{2}=\rho^{-n}, \quad Z_{3}=J_{n}(\sqrt{\mathfrak{F}} \rho), \quad Z_{4}=Y_{n}(\sqrt{\mathfrak{F}} \rho)
$$

where $c_{1}, c_{2}, c_{3}$ and $c_{4}$ are integration constants. Otherwise, if the plate is an annular one we use a numerical algorithm (for example the Runge-Kutta method) in the solution procedure - see 37] for details.

The physical quantities in the plate can also be expanded into a Fourier series similarly to the deflection. Thus we get

$$
\vartheta=\vartheta_{o}+\sum_{m=0}^{1} \sum_{n=1}^{\infty} \vartheta_{n}^{m} \cos \left(n \varphi-m \frac{\pi}{2}\right),
$$

for the rotation field,

$$
M_{R}=M_{R o}+\sum_{m=0}^{1} \sum_{n=1}^{\infty} \stackrel{m}{M}_{R n} \cos \left(n \varphi-m \frac{\pi}{2}\right),
$$

for the bending moment, and

$$
Q_{R}=Q_{R o}+\sum_{m=0}^{1} \sum_{n=1}^{\infty} \stackrel{m}{Q}_{R n} \cos \left(n \varphi-m \frac{\pi}{2}\right)
$$

for the shear force.

It can be shown that the amplitude functions of the rotation $\psi_{\varphi}$, the bending moment $M_{R}$ and the shear force $Q_{R}$ can all be given in terms of the amplitudes of $w$ as follows:

$$
\begin{gathered}
\stackrel{m}{\vartheta}_{n}=-\frac{1}{R_{e}} \frac{\mathrm{d} w_{n}}{\mathrm{~d} \rho}, \\
\stackrel{m}{M}_{R n}=-\frac{I_{1} E_{1 p}}{R_{e}^{2}}\left[\frac{\mathrm{d}^{2} \stackrel{m}{w}_{n}}{\mathrm{~d} \rho^{2}}+\frac{\nu}{\rho}\left(\frac{\mathrm{d} w_{n}}{\mathrm{~d} \rho}-\frac{n^{2}}{\rho}{ }_{w_{n}}\right)\right] \\
\stackrel{m}{Q}_{R n}=\frac{I_{1 p} E_{1 p}}{R_{e}^{3}}\left[\frac{\mathrm{d}^{3} \stackrel{m}{w}_{n}}{\mathrm{~d} \rho^{3}}+\frac{1}{\rho} \frac{\mathrm{d}^{2} m_{n}^{m}}{\mathrm{~d} \rho^{2}}-\left(\frac{1}{\rho^{2}}\left(1+2 n^{2}(1-\nu)\right)+\mathfrak{F} \mathcal{N}_{R}\right) \frac{\mathrm{d} w_{n}}{\mathrm{~d} \rho}+\frac{3 n^{2}}{\rho^{3}} \stackrel{m}{w}_{n}\right] .
\end{gathered}
$$

\subsection{Governing equations for the cylindrical shell.}

3.3.1. Field eqations for the shell. The kinematic quantities which describe the deformations of the shell can be calculated by the displacements $u_{\varphi}, u_{\xi}$ and $u_{\zeta}$. Using the coordinate $\xi$ the rotations can be obtained from the relations

$$
\begin{aligned}
\psi_{\varphi} & =\frac{1}{R_{e}} \frac{\partial u_{\zeta}}{\partial \xi} \\
\psi_{x} & =\frac{1}{R_{e}}\left(\frac{\partial u_{\zeta}}{\partial \varphi}-u_{\varphi}\right)
\end{aligned}
$$


The deformations on the middle surface of the shell are also characterized by the axial strains

$$
\begin{aligned}
e_{x x} & =\frac{1}{R_{e}} \frac{\partial u_{\xi}}{\partial \xi} \\
e_{x \varphi} & =\frac{1}{2 R_{e}}\left(\frac{\partial u_{\xi}}{\partial \varphi}+\frac{\partial u_{\varphi}}{\partial \xi}\right), \\
e_{\varphi \varphi} & =\frac{1}{R_{e}}\left(\frac{\partial u_{\varphi}}{\partial \varphi}+u_{\zeta}\right)
\end{aligned}
$$

and by the elements of the curvature tensor:

$$
\begin{aligned}
\kappa_{x x} & =-\frac{1}{R_{e}^{2}} \frac{\partial^{2} u_{\zeta}}{\partial \xi^{2}}, \\
\kappa_{\varphi \varphi} & =-\frac{1}{R_{e}^{2}} \frac{\partial^{2} u_{\zeta}}{\partial \varphi^{2}}, \\
\kappa_{x \varphi} & =\kappa_{\varphi x}=-\frac{1}{R_{e}^{2}} \frac{\partial^{2} u_{\zeta}}{\partial \xi \partial \varphi} .
\end{aligned}
$$

Using these kinematic quantities the corresponding inner forces are obtained from Hooke's law:

$$
\begin{aligned}
& N_{x x}=E_{1 s} b_{s}\left(e_{x x}+\nu e_{\varphi \varphi}\right), \\
& N_{\varphi \varphi}=E_{1 s} b_{s}\left(e_{\varphi \varphi}+\nu e_{x x}\right), \\
& N_{\varphi x}=E_{1 s} b_{s}(1-\nu) e_{x \varphi} .
\end{aligned}
$$

The elements of the internal moments tensor are given by the relations below:

$$
\begin{aligned}
& M_{x x}=E_{1 s} I_{1 s}\left(\kappa_{x x}+\nu \kappa_{\varphi \varphi}\right), \\
& M_{\varphi \varphi}=E_{1 s} I_{1 s}\left(\kappa_{\varphi \varphi}+\nu \kappa_{x x}\right), \\
& M_{x \varphi}=E_{1 s} I_{1 s}(1-\nu) \kappa_{x \varphi} .
\end{aligned}
$$

Note that the internal moment tensor is assumed to be symmetric, i.e. $M_{x \varphi}=M_{\varphi x}$.

The shear forces on the middle surface of the shell are denoted by $Q_{x \zeta}$ and $Q_{\varphi \zeta}$. The above equations are associated with the equilibrium equations

$$
\begin{aligned}
\frac{1}{R_{e}} \frac{\partial N_{x x}}{\partial \xi}+\frac{1}{R_{e}} \frac{\partial N_{\varphi x}}{\partial \varphi}+p_{x} & =0 \\
\frac{1}{R_{e}} \frac{\partial N_{\varphi x}}{\partial \xi}+\frac{1}{R_{e}} \frac{\partial N_{\varphi \varphi}}{\partial \varphi}+\frac{1}{R_{e}} Q_{\varphi \zeta}+p_{\varphi} & =0 \\
-\frac{1}{R_{e}} \frac{\partial Q_{x \zeta}}{\partial \xi}-\frac{1}{R_{e}} \frac{Q_{\varphi \zeta}}{\partial \varphi}-\frac{1}{R_{e}} N_{\varphi \varphi}+p_{\zeta} & =0 \\
\frac{1}{R_{e}} \frac{\partial M_{x x}}{\partial \xi}+\frac{1}{R_{e}} \frac{\partial M_{\varphi x}}{\partial \varphi}+Q_{x \zeta} & =0 \\
\frac{1}{R_{e}} \frac{\partial M_{x \varphi}}{\partial \xi}+\frac{1}{R_{e}} \frac{\partial M_{\varphi \varphi}}{\partial \varphi}+Q_{\varphi \zeta} & =0
\end{aligned}
$$


in which $p_{x}, p_{\varphi}$ and $p_{\zeta}$ are the intensities of the distributed loads exerted on the middle surface of the shell.

Observe that we have as many equations as there are unknowns (nineteen equations (3.17)-3.22 in nineteen unknowns).

3.3.2. The Galerkin function. For $p_{x}=p_{\varphi}=0$ the fundamental equations (obtained after we have eliminated the intermediate variables) set up for the displacement coordinates $u_{x i}, u_{\varphi}$ and $u_{\zeta}$ will be fulfilled identically if we calculate the displacement coordinates in terms of the Galerkin function $\phi(\xi, \varphi)$ using the relations 35, 36

$$
\begin{aligned}
& u_{\xi}=\frac{\partial^{3} \phi}{\partial \xi \partial \varphi^{2}}-\nu_{s} \frac{\partial^{3} \phi}{\partial \xi^{3}}, \\
& u_{\varphi}=-\frac{\partial^{3} \phi}{\partial \varphi^{3}}-\left(2+\nu_{s}\right) \frac{\partial^{3} \phi}{\partial \xi^{2} \partial \varphi}, \\
& u_{\zeta}=\nabla^{2} \nabla^{2} \phi
\end{aligned}
$$

in which $\phi$ should satisfy the differential equation

$$
\nabla^{2} \nabla^{2} \nabla^{2} \nabla^{2} \phi+4 \hat{\beta^{4}} \frac{\partial^{4} \phi}{\partial \xi^{4}}=\frac{4 \hat{\beta}^{4} R_{e}^{2}}{E_{s} b_{s}} p_{\zeta}
$$

where

$$
4 \hat{\beta^{4}}=12\left(1-\nu_{s}^{2}\right) \frac{R_{e}^{2}}{b_{s}^{2}}
$$

and

$$
\nabla^{2}=\frac{\partial^{2}}{\partial \xi^{2}}+\frac{\partial^{2}}{\partial \varphi^{2}}
$$

Every physical quantity appearing in the boundary conditions can be written in terms of the Galerkin function $\phi$ if we substitute the displacements 3.23 into equations (3.17)-3.22. Omitting the formal transformations we get:

$$
\begin{aligned}
\psi_{\varphi} & =\frac{1}{R_{e}} \frac{\partial}{\partial \xi} \nabla^{2} \nabla^{2} \phi \\
N_{x x} & =\frac{E_{s} b_{s}}{R_{e}} \frac{\partial^{4} \phi}{\partial \xi^{2} \partial \varphi^{2}}, \\
N_{\varphi x} & =-\frac{E_{s} b_{s}}{R_{e}} \frac{\partial^{4} \phi}{\partial \xi^{3} \partial \varphi}, \\
M_{x x} & =-\frac{E_{s} b_{s}}{4 \hat{\beta}^{4}}\left[\frac{\partial^{2}}{\partial \xi^{2}}+\nu_{s} \frac{\partial^{2}}{\partial \varphi^{2}}\right] \nabla^{2} \nabla^{2} \phi, \\
M_{x \varphi} & =-\frac{E_{s} b_{s}\left(1-\nu_{s}\right)}{4 \hat{\beta}^{4}} \frac{\partial^{2}}{\partial \xi \partial \varphi} \nabla^{2} \nabla^{2} \phi, \\
Q_{x \zeta} & =\frac{1}{R_{e}} \frac{E_{s} b_{s}}{4 \hat{\beta^{4}}} \frac{\partial}{\partial \xi} \nabla^{2} \nabla^{2} \nabla^{2} \phi .
\end{aligned}
$$


3.3.3. Solution for the Galerkin function. Let us expand the function $\phi(\xi, \varphi)$ into a Fourier series similarly to the deflection $w$. We can write

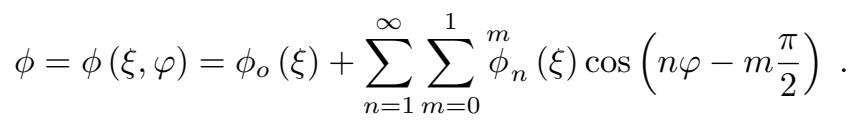

Substituting the series 3.27 into differential equation 3.24 we obtain that the coefficients ${ }_{n}$ of the series should satisfy the ordinary differential equations

$$
\begin{gathered}
\frac{\mathrm{d}^{8} \stackrel{m}{\phi}_{n}}{\mathrm{~d} \xi^{8}}-4 n^{2} \frac{\mathrm{d}^{6} \stackrel{m}{\phi}_{n}}{\mathrm{~d} \xi^{6}}+6 n^{4} \frac{\mathrm{d}^{4} \phi_{n}}{\mathrm{~d} \xi^{4}}-4 n^{6} \frac{\mathrm{d}^{2} \phi_{n}}{\mathrm{~d} \xi^{2}}+n^{8}{\stackrel{m}{\phi_{n}}}+4 \hat{\beta}^{4} \frac{\mathrm{d}^{4} \phi_{n}}{\mathrm{~d} \xi^{4}}=\frac{4 \hat{\beta}^{4} R_{e}^{2}}{E b_{s}}{\stackrel{m}{p_{\zeta n}}}_{m=0,1 ; \quad n=0,1,2, \ldots} \\
m=0
\end{gathered}
$$

where the coefficients $\stackrel{m}{p}_{\zeta n}$ on the right side are those of the Fourier series that belongs to the load exerted on the middle surface of the shell:

$$
p_{\zeta}(\xi, \varphi)=p_{\zeta o}(\xi)+\sum_{n=1}^{\infty} \sum_{m=0}^{1} \stackrel{m}{p}_{\zeta n}(\xi) \cos \left(n \varphi-m \frac{\pi}{2}\right)
$$

In the investigated problem this load is zero, consequently the solutions of differential equations 3.28 have only a homogeneous part.

The solution of equation 3.28 is sought in the form

$$
\stackrel{m}{\phi_{n}}=e^{\lambda_{n} \xi}
$$

After substituting it into equation 3.28 we get the characteristic polynomial for the $(m, n)$-th differential equation:

$$
\left(\lambda_{n}^{2}-n^{2}\right)^{4}=-4 \hat{\beta}^{4} \lambda_{n}^{4}
$$

By introducing the notations

$$
\begin{aligned}
& b_{n}=\frac{\hat{\beta}}{2} \sqrt{\sqrt{1+4\left(\frac{n}{\hat{\beta}}\right)^{4}}+2\left(\frac{n}{\hat{\beta}}\right)^{2}}, \\
& a_{n}=\frac{\hat{\beta}}{2} \sqrt{\sqrt{1+4\left(\frac{n}{\hat{\beta}}\right)^{4}}-2\left(\frac{n}{\hat{\beta}}\right)^{2}},
\end{aligned}
$$

and

$$
\begin{aligned}
& \beta_{n 1}=b_{n}+\frac{\hat{\beta}}{2}, \\
& \beta_{n 2}=b_{n}-\frac{\hat{\beta}}{2}, \\
& \alpha_{n 1}=\frac{\hat{\beta}}{2}+a_{n},
\end{aligned}
$$




$$
\alpha_{n 2}=\frac{\hat{\beta}}{2}-a_{n}
$$

it can be shown that the roots of equation (3.31) are as follows:

$$
\begin{aligned}
& \lambda_{n 1}=-\beta_{n 2}+i \alpha_{n 2}, \\
& \lambda_{n 2}=-\beta_{n 1}+i \alpha_{n 1}, \\
& \lambda_{n 3}=-\beta_{n 2}-i \alpha_{n 2}, \\
& \lambda_{n 4}=-\beta_{n 1}-i \alpha_{n 1}, \\
& \lambda_{n 5}=-\lambda_{n 1}, \\
& \lambda_{n 6}=-\lambda_{n 2}, \\
& \lambda_{n 7}=-\lambda_{n 3}, \\
& \lambda_{n 8}=-\lambda_{n 4} .
\end{aligned}
$$

Substituting solution 3.34 into 3.30 and making some manipulations we get the real part of the solution for the Galerkin function:

$$
\begin{aligned}
\stackrel{m}{\phi}_{n}=\sum_{j=1}^{2}\left\{\stackrel{m}{K}_{n j}\right. & \sinh \left(\beta_{n j} \xi\right) \sin \left(\alpha_{n j} \xi\right)+\stackrel{m}{M}_{n j} \sinh \left(\beta_{n j} \xi\right) \cos \left(\alpha_{n j} \xi\right)+ \\
& \left.+\stackrel{m}{P}_{n j} \cosh \left(\beta_{n j} \xi\right) \sin \left(\alpha_{n j} \xi\right)+\stackrel{m}{S}_{n j} \cosh \left(\beta_{n j} \xi\right) \cos \left(\alpha_{n j} \xi\right)\right\}
\end{aligned}
$$

where $\stackrel{m}{K}_{n j}, \stackrel{m}{M}_{n j}, \stackrel{m}{P}_{n j}$ and $\stackrel{m}{S}_{n j}$ constitute eight undetermined integration constants.

The Fourier series of the physical quantities in the shell can be written in a form similar to the series 3.11:

$$
\begin{aligned}
u_{\xi} & =u_{\xi o}+\sum_{n=1}^{\infty} \sum_{m=0}^{1}{\stackrel{m}{u_{\xi n}}}_{m} \cos \left(n \varphi-m \frac{\pi}{2}\right) \\
u_{\varphi} & =u_{\varphi o}+\sum_{n=1}^{\infty} \sum_{m=0}^{1} \stackrel{m}{u}_{\varphi n} \sin \left(n \varphi-m \frac{\pi}{2}\right) \\
u_{\zeta} & =u_{\zeta o}+\sum_{n=1}^{\infty} \sum_{m=0}^{1} \stackrel{m}{u}_{\zeta n} \cos \left(n \varphi-m \frac{\pi}{2}\right) \\
\psi_{\varphi} & =\psi_{\varphi o}+\sum_{n=1}^{\infty} \sum_{m=0}^{1} \stackrel{m}{\psi}_{\varphi n} \cos \left(n \varphi-m \frac{\pi}{2}\right) \\
N_{x x} & =N_{x x o}+\sum_{n=1}^{\infty} \sum_{m=0}^{1} \stackrel{m}{N}_{x x n} \cos \left(n \varphi-m \frac{\pi}{2}\right) \\
N_{\varphi x} & =N_{\varphi x o}+\sum_{n=1}^{\infty} \sum_{m=0}^{1} \stackrel{m}{N}_{\varphi x n} \sin \left(n \varphi-m \frac{\pi}{2}\right)
\end{aligned}
$$




$$
\begin{aligned}
& M_{x x}=M_{x x o}+\sum_{n=1}^{\infty} \sum_{m=0}^{1} \stackrel{m}{M}_{x x n} \cos \left(n \varphi-m \frac{\pi}{2}\right), \\
& M_{x \varphi}=M_{x \varphi o}+\sum_{n=1}^{\infty} \sum_{m=0}^{1} \stackrel{m}{M}_{x \varphi n} \sin \left(n \varphi-m \frac{\pi}{2}\right), \\
& Q_{x \zeta}=Q_{x \zeta o}+\sum_{n=1}^{\infty} \sum_{m=0}^{1} \stackrel{m}{Q}_{x \zeta n} \cos \left(n \varphi-m \frac{\pi}{2}\right) .
\end{aligned}
$$

Utilizing relations $(3.23)$ and $(3.26)$, which provide the physical quantities on the middle surface of the shell in terms of the Galerkin function, the coefficients in the series 3.36 can also be given in terms of the coefficients ${ }_{\phi}{ }_{n}$ of the Galerkin functions

$$
\begin{aligned}
& \stackrel{m}{u}_{\xi n}=-\left(n^{2} \frac{\mathrm{d} \phi_{n}}{\mathrm{~d} \xi}+\nu \frac{\mathrm{d}^{3} \phi_{n}}{\mathrm{~d} \xi^{3}}\right), \\
& \stackrel{m}{u}_{\varphi n}=\left(-n^{3} \stackrel{m}{\phi}_{n}+n(2+\nu) \frac{\mathrm{d}^{2} \phi_{n}^{m}}{\mathrm{~d} \xi^{2}}\right) \text {, } \\
& {\stackrel{m}{u_{\zeta n}}}=\left(n^{4} \phi_{n}^{m}-2 n^{2} \frac{\mathrm{d}^{2} \phi_{n}^{m}}{\mathrm{~d} \xi^{2}}+\frac{\mathrm{d}^{4} \phi_{n}}{\mathrm{~d} \xi^{4}}\right), \\
& \stackrel{m}{\psi}_{\phi n}=-\frac{1}{R_{e}}\left(n^{4} \frac{\mathrm{d} \phi_{n}}{\mathrm{~d} \xi}-2 n^{2} \frac{\mathrm{d}^{3} \phi_{n}}{\mathrm{~d} \xi^{3}}+\frac{\mathrm{d}^{5} \phi_{n}^{m}}{\mathrm{~d} \xi^{5}}\right), \\
& \stackrel{m}{N}_{x x n}=-\frac{E_{s} b_{s}}{R_{e}} n^{2} \frac{\mathrm{d}^{2} \phi_{n}}{\mathrm{~d} \xi^{2}}, \\
& \stackrel{m}{N}_{\varphi x n}=-\frac{E_{s} b_{s}}{R_{e}} n \frac{\mathrm{d}^{3} \phi_{n}^{m}}{\mathrm{~d} \xi^{3}}, \\
& \stackrel{m}{M}_{x x n}=-\frac{E_{s} b_{s}}{4 \hat{\beta}^{4}}\left(n^{4} \frac{\mathrm{d}^{2} \stackrel{m}{\phi}_{n}}{\mathrm{~d} \xi^{2}}-2 n^{2} \frac{\mathrm{d}^{4} \phi_{n}}{\mathrm{~d} \xi^{4}}+\frac{\mathrm{d}^{6} \stackrel{m}{\phi}_{n}}{\mathrm{~d} \xi^{6}}-\nu n^{2}\left[n^{4} \phi_{n}-2 n^{2} \frac{\mathrm{d}^{2} \phi_{n}^{m}}{\mathrm{~d} \xi^{2}}+\frac{\mathrm{d}^{4} \phi_{n}}{\mathrm{~d} \xi^{4}}\right]\right), \\
& \stackrel{m}{M}_{x \varphi n}=\frac{(1-\nu) E_{s} b_{s}}{4 \hat{\beta}^{4}}\left(n^{5} \frac{\mathrm{d} \phi_{n}}{\mathrm{~d} \xi}-2 n^{3} \frac{\mathrm{d}^{3} \phi_{n}^{m}}{\mathrm{~d} \xi^{3}}+n \frac{\mathrm{d}^{5} \phi_{n}}{\mathrm{~d} \xi^{5}}\right) \\
& \stackrel{m}{Q}_{x \zeta n}=\frac{1}{R_{e}} \frac{E_{s} b_{s}}{4 \hat{\beta}^{4}}\left(-n^{6} \frac{\mathrm{d} \phi_{n}}{\mathrm{~d} \xi}+3 n^{4} \frac{\mathrm{d}^{3} \stackrel{m}{\phi}_{n}}{\mathrm{~d} \xi^{3}}-3 n^{2} \frac{\mathrm{d}^{5} \dot{m}_{n}}{\mathrm{~d} \xi^{5}}+\frac{\mathrm{d}^{7} \stackrel{m}{\phi}_{n}}{\mathrm{~d} \xi^{7}}\right) .
\end{aligned}
$$


Substituting equation 3.35 into the above relations we obtain the Fourier coefficients of the physical quantities appearing in the boundary conditions in terms of the coordinate $\xi$. For the sake of brevity these are omitted here.

3.4. Boundary- and continuity conditions. A solution for the amplitude of the displacement field on the middle surface of the plate contains four, while a solution for $\stackrel{m}{\phi_{n}}$ involves eight integration constants. The stiffening shell is mentally divided in two separate shells at the intersection line of the middle surfaces of the plate and the shell. Therefore we need two solutions for each of the two parts of the shell, consequently we have to determine altogether twenty integration constants.

In what follows we shall present the boundary- and continuity conditions which provide the integration constants. We begin with the free boundaries of the shell, where the following conditions should be satisfied:

$$
\begin{aligned}
& \stackrel{m}{N}_{x x n}\left(\xi= \pm \xi_{h}\right)=0, \\
& \stackrel{m}{N}_{x \varphi n}\left(\xi= \pm \xi_{h}\right)+n \stackrel{m}{M}_{x \varphi n}\left(\xi= \pm \xi_{h}\right) / R_{e}=0 \text {, } \\
& \stackrel{m}{M}_{x x n}\left(\xi= \pm \xi_{h}\right)=0, \\
& \stackrel{m}{Q}_{x \zeta n}\left(\xi= \pm \xi_{h}\right)-n \stackrel{m}{M}_{x \varphi n}\left(\xi= \pm \xi_{h}\right) / R_{e}=0 .
\end{aligned}
$$

These are altogether eight equations.

The shell and plate deform together on the intersection line of the middle surfaces of the shell and the plate, which results in the following kinematic continuity conditions:

$$
\begin{aligned}
& \stackrel{m}{u}_{\xi n}(\xi= \pm 0)=\stackrel{m}{w}_{n}(\rho=1) \text {, } \\
& \stackrel{m}{\psi}_{\varphi n}(\xi= \pm 0)=\stackrel{m}{\vartheta}_{n}(\rho=1) \text {, } \\
& \stackrel{m}{u}_{\zeta n}(\xi= \pm 0)=\stackrel{m}{u}_{n}(\rho=1)=0 \text {, } \\
& \stackrel{m}{u}_{\varphi n}(\xi= \pm 0)=\stackrel{m}{v}_{n}(\rho=1)=0 \text {, } \\
& n \neq 0
\end{aligned}
$$

Here the two sides of the plate at $\xi=0$ are designated by $\xi=+0$ and $\xi=-0$. Observe that conditions $\sqrt{3.39 \mathrm{c}} \mathrm{d})$ reflect the fact that the plane stress problem is axisymmetric, i.e. condition (3.1) together with (3.4), furthermore $\stackrel{m}{v}_{o}(\rho=1)=\stackrel{m}{u}_{\varphi o}(\xi=0)$ holds if $n=0$. The kinematic continuity conditions provide eight independent equations.

For the shear force $\stackrel{m}{Q}_{x \zeta n}$ we can not prescribe any condition, since $\stackrel{m}{u}_{\zeta n}(\xi=0)=0$. Since $\stackrel{m}{u}_{\varphi n}(\rho=1)=0$ we can not prescribe continuity conditions for the inner forces $\stackrel{m}{N}_{R \varphi n}$ and $\stackrel{m}{N}_{x \varphi n}$. However the axisymmetric parts of these quantities are also equal to zero.

It follows from the global equilibrium of the structure that the axisymmetric part of the shear force should meet the condition $Q_{R o}=0$. Otherwise the continuity 
condition

$$
\stackrel{m}{Q}_{R n}(\rho=1)+\stackrel{m}{N}_{x x n}(\xi=+0)-\stackrel{m}{N}_{x x n}(\xi=-0)=0
$$

should be fulfilled - see Figure 5 a.

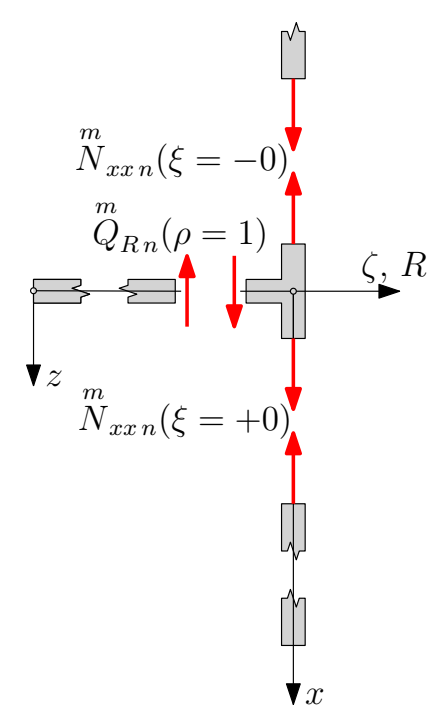

a.

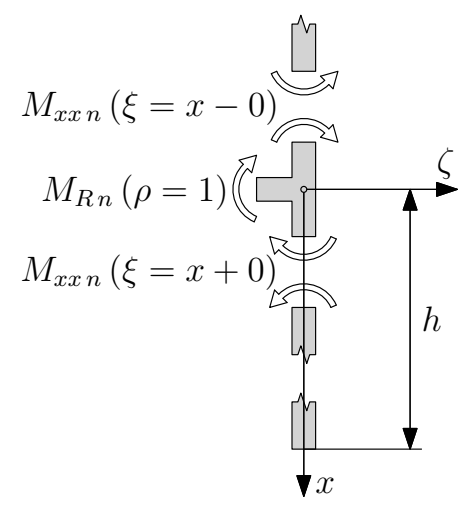

b.

Figure 5. Forces and moments between the two elements

As regards the bending moments, equation

$$
\stackrel{m}{M}_{R n}(\rho=1)+\stackrel{m}{M}_{x x n}(\xi=+0)-\stackrel{m}{M}_{x x n}(\xi=-0)=0
$$

is the continuity condition for the coefficients of the Fourier series - see Figure 5b.

The boundary conditions on the inner boundary of the plate depend on the supports applied. It is clear that two boundary conditions can be prescribed on the inner boundary. Consequently the boundary- and continuity conditions provide altogether twenty homogenous equations for the twenty integration constants. These equations involve $\mathfrak{F}$ as a parameter. Therfore the critical value of $\mathfrak{F}$ can be determined from the condition that the system determinant should vanish.

\section{NumericAl RESUlts}

4.1. Circular plate. If the plate has no hole in it then the displacement $\stackrel{m}{w}$ and its derivatives (i.e. the rotation $\psi_{\varphi n}$ ) have to be finite:

$$
\begin{aligned}
& \stackrel{m}{w}_{n}=\text { finite } \\
& \psi_{\varphi n}=\text { finite } .
\end{aligned}
$$


A code has been written in Fortran 90 to solve the non-linear equation for $\mathfrak{F}_{c r}$ and compute $\mathfrak{F}_{\text {ocr }}$. The calculations were made with the material and thickness properties taken as the same $\left(E=E_{p}=E_{s}=200 \mathrm{GPa}, \nu=\nu_{p}=\nu_{s}=0.3, b=b_{p}=b_{s}\right)$ and the thickness to radius ratio had a fixed value $\left(b / R_{e}=0.01\right)$. The computational results are presented in Figure 6 for the first 4 members of the Fourier-series and for axisymmetric deformations $(n=0)$. It is clear from the graphs that the height of the plate does not affect the critical load if the height is larger than a certain value. One can see that the lowest value of the critical load belongs to the case of the axisymmetric deformation; the only exception is the critical load if $n=2$ and the shell height (i.e. the $\frac{h}{R_{e}}$ ratio) is small.

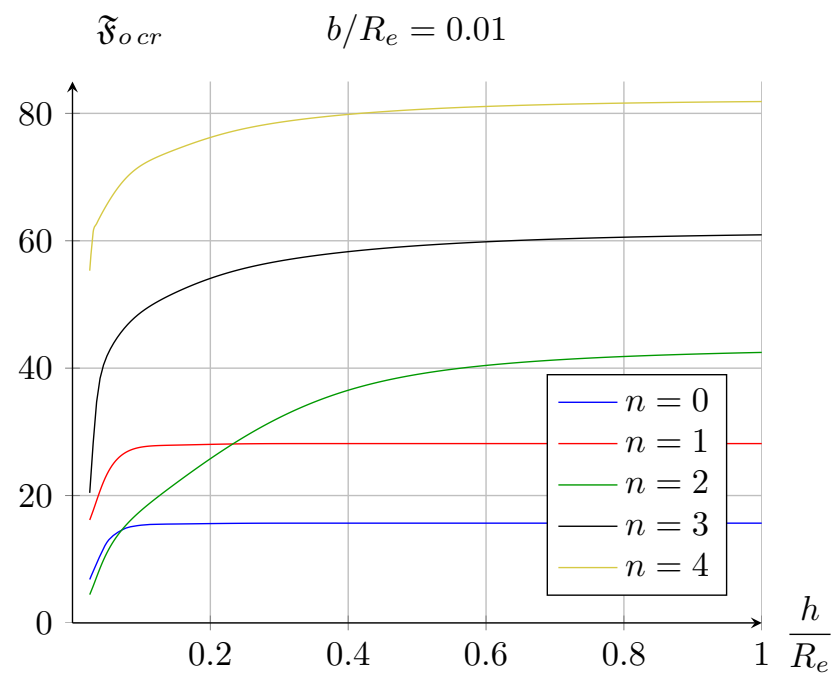

Figure 6. Critical load for a circular plate

4.2. Annular plate with simple support. Consider a stiffened annular plate with simple support on its inner boundary -see Figure 2 c. The boundary conditions at the inner edge are as follows:

$$
\begin{gathered}
\stackrel{m}{w_{n}}\left(\rho=\rho_{i}\right)=0, \\
\stackrel{m}{M}_{R n}\left(\rho=\rho_{i}\right)=0 .
\end{gathered}
$$

Figure 7 shows the buckling load parameter $\mathfrak{F}$ versus the $\frac{h}{R_{e}}$ height of the shell in three diagrams if the inner radii are $\rho_{i}=0.25, \rho_{i}=0.5$ and $\rho_{i}=0.75$. The other data were the same as in the previous problem. The curves are all asymptotic in a different measure. The smallest critical load belongs to axisymmetric deformations, but if the height is relatively small the curve belonging to $n=2$ has smaller values. This curve has also the slowest convergence. The range where asymmetric mode gives the buckling load increases if the inner radius becomes greater. 


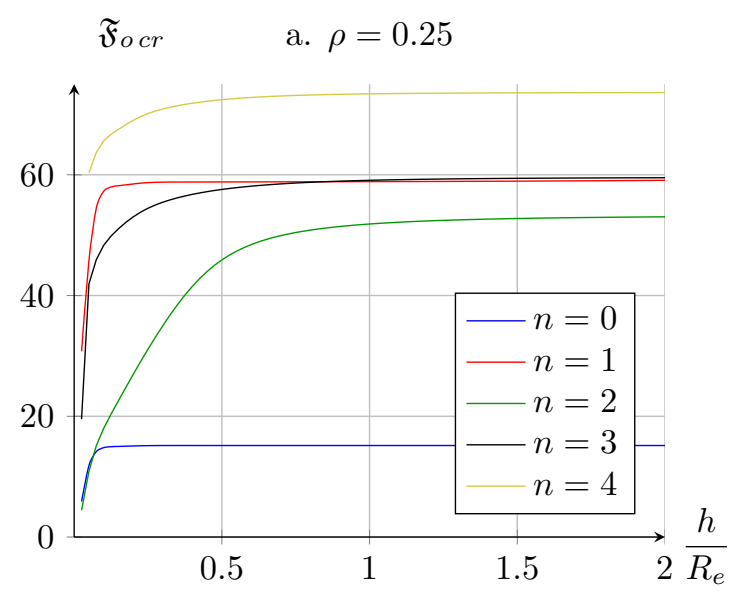

$\mathfrak{F}_{\text {ocr }} \quad$ b. $\rho=0.5$

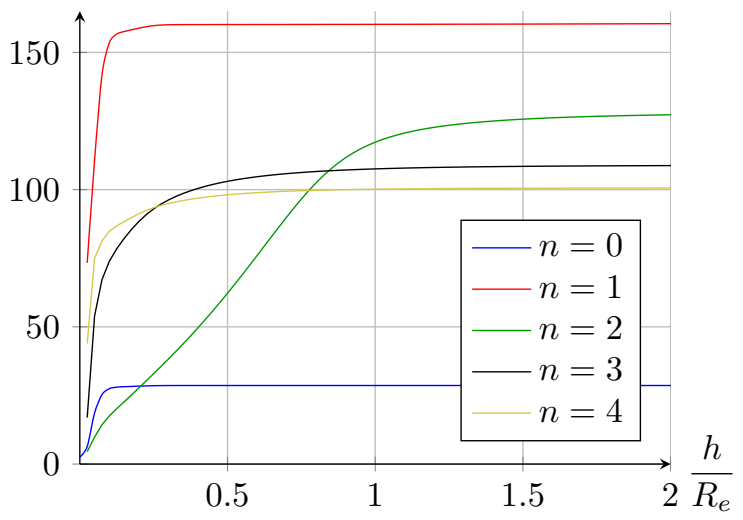

$\mathfrak{F}_{o c r} \quad$ c. $\rho=0.75$

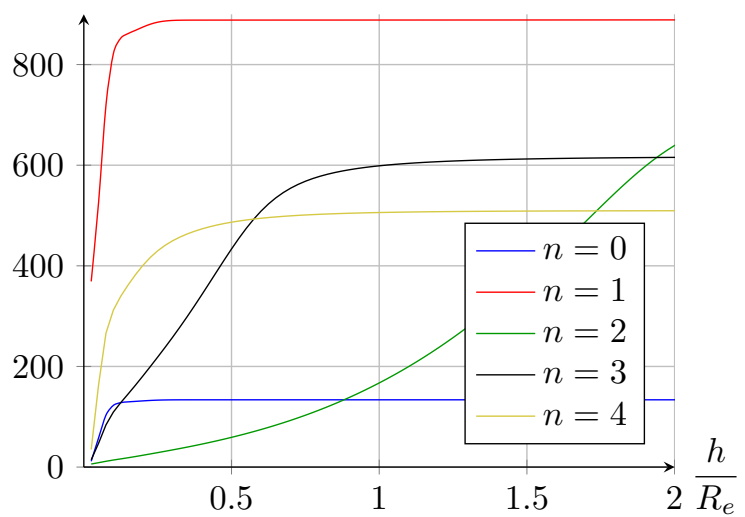

Figure 7. Critical load of simply supported annular plates 
Figure 8 shows the critical load against $\rho_{i}$ and $\frac{h}{R_{e}}$ for the modes $n=1, \ldots, 4$. The axis $\mathfrak{F}_{\text {o cr }}$ is a logarithmic one.

a. $n=1$

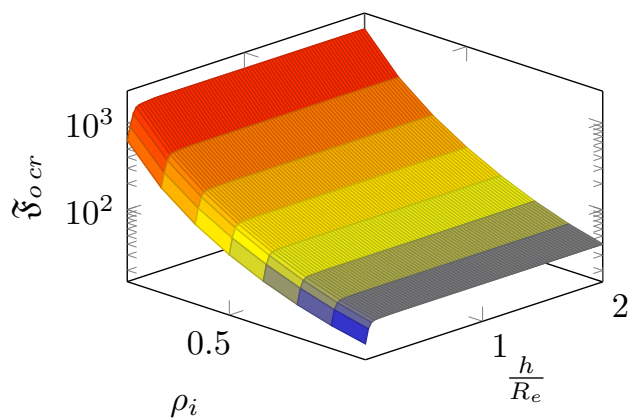

c. $n=3$

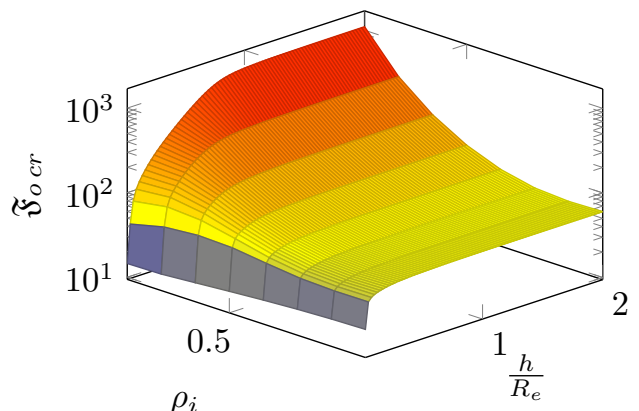

b. $n=2$

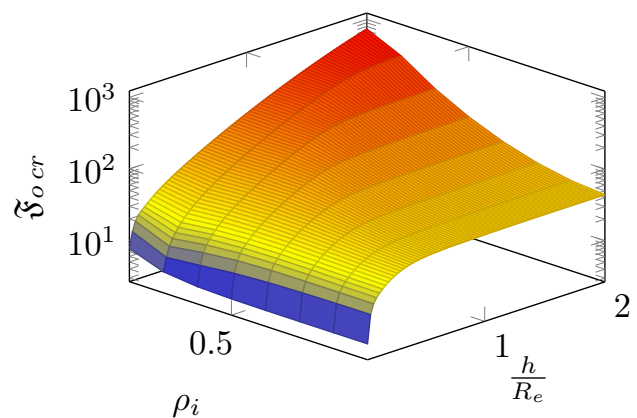

d. $n=4$

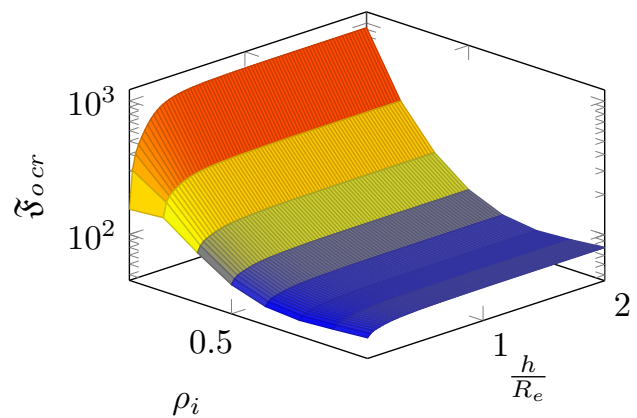

Figure 8. Critical load against $\rho_{i}$ and $h / R_{e}$

4.3. Annular plate with clamped inner boundary. If (a) the radial displacement is not prescribed on the inner boundary but (b) the rotation is zero (in contrast to the previous problem) - see Fig. $2 \mathrm{~b}$. for further details - then the equation

$$
\stackrel{m}{\vartheta}_{n}\left(\rho=\rho_{i}\right)=0
$$

together with equation $4.2 \mathrm{a}$ are the boundary conditions on the inner boundary.

Figure 9 shows the critical load $\mathfrak{F}_{o c r}$ against the height of the shell (i.e. $\frac{h}{R_{e}}$ ). The curves in the diagrams have a similar progress to the ones presented in the previous problem. AS the inner radius is getting greater, the interval where asymmetric deformations give the lowest critical load increases. Doing the calculations for higher numbers of $n$ also the asymptotic values of the critical load approach values belonging to axisymmetric deformations. In some cases its even smaller - see Figure 10, where the critical load is shown against the inner radius $\rho_{i}$. The height of the stiffening shell is kept constant $\left(\frac{h}{R_{e}}=2\right)$. 


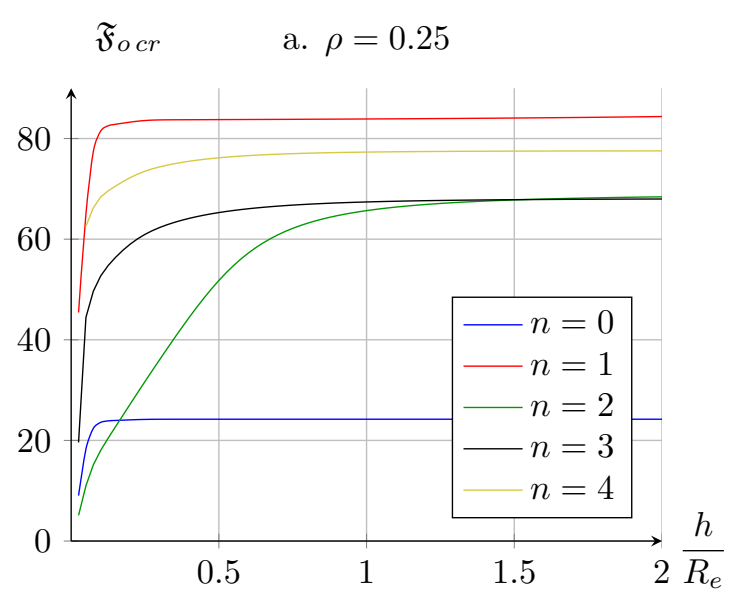

$\mathfrak{F}_{\text {ocr }}$

b. $\rho=0.5$

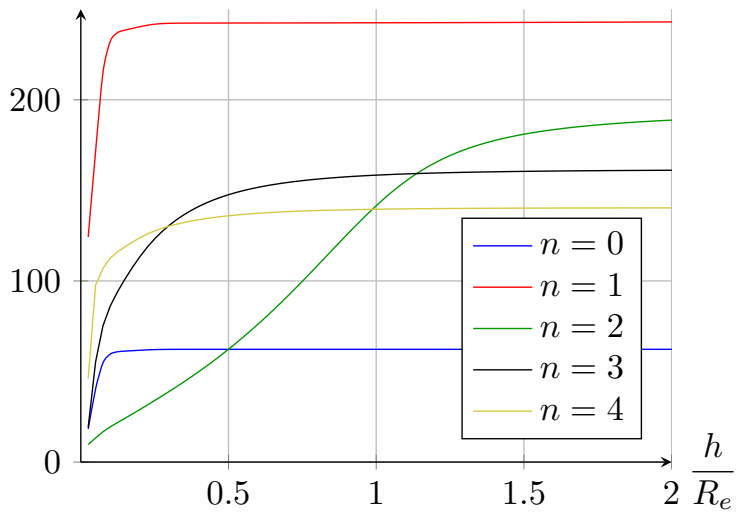

$\mathfrak{F}_{\text {ocr }}$

c. $\rho=0.75$

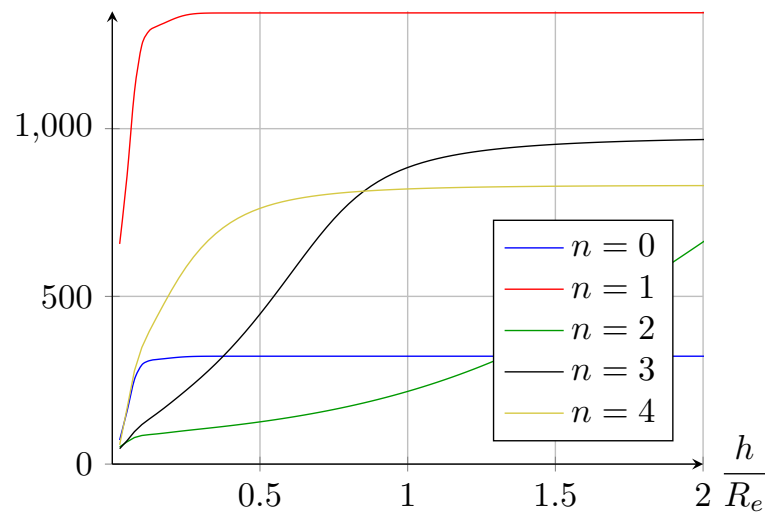

Figure 9. Critical load of plates with clamped inner boundary 


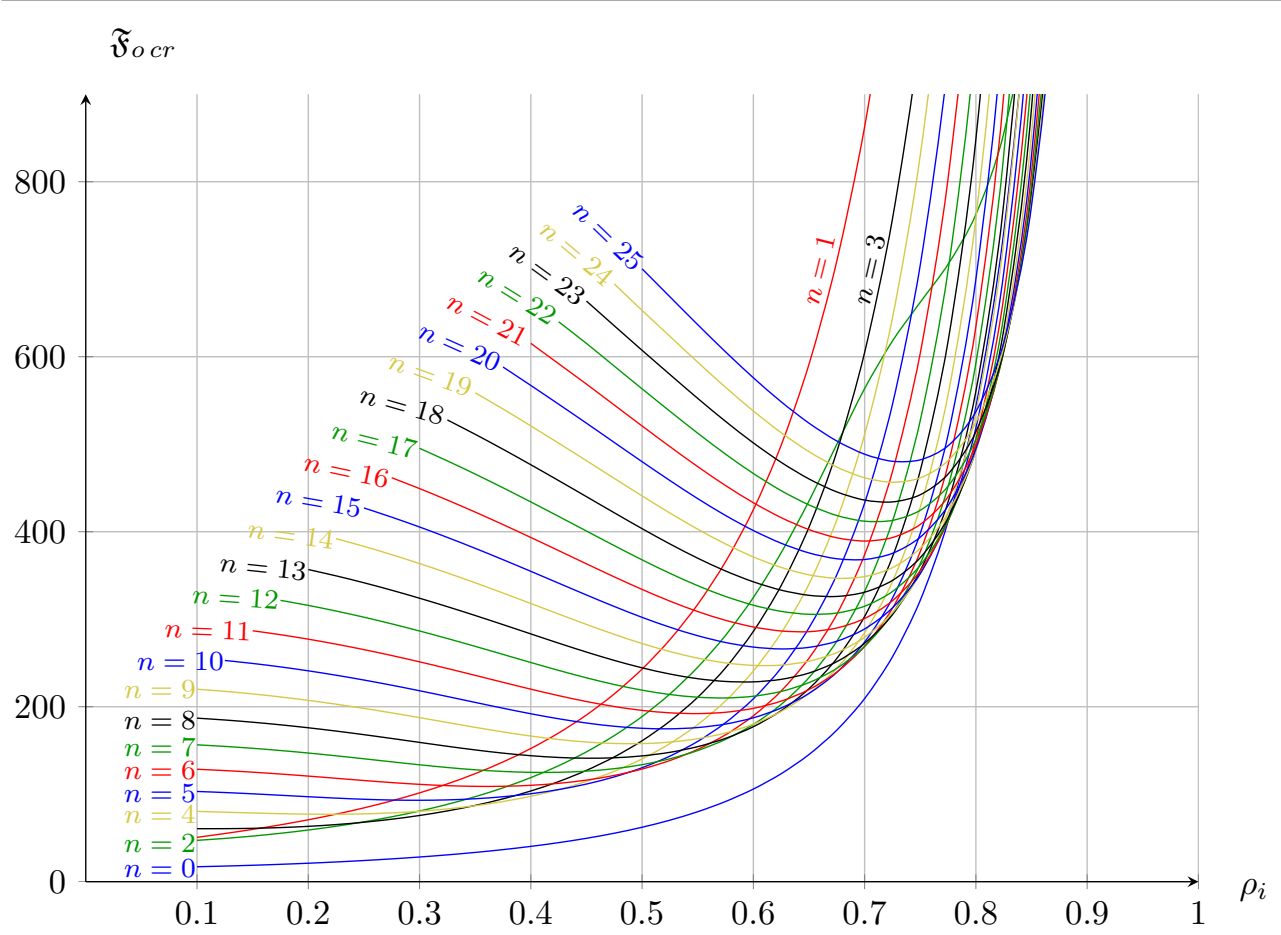

Figure 10. Critical load versus inner radius

\section{Concluding Remarks}

The present paper has established the equations that can be used to determine the critical load of circular and annular plates stiffened on the outer edge by a cylindrical shell under the assumption of non-axisymmetric deformations. We have clarified what the continuity conditions are between the two separate elements of the structure. We have also presented the solutions for the critical load of circular and annular plates with two different types of support assuming axisymmetric and non-axisymmetric deformations. It is obvious from the results that the stiffening significantly increases the critical load.It has been observed that buckling load belongs to axisymmetric mode if the plate is circular. Non-axisymmetric buckling modes can be observed if the geometric properties (inner radius, shell height) are in a certain range.

Acknowledgements. This research was (partially) carried out in the framework of the Center of Excellence of Innovative Engineering Design and Technologies at the University of Miskolc.

\section{REFERENCES}

1. BRYAN, G. H.: On the stability of a plate under thrust in its own plane with applications to the buckling of the sides of a ship. Proceedings of the London Mathematical Society, (1890), 54-67. 
2. NÁdAI, A.: Über das Ausbeulen von Kreisförmingen Platten. Zeitschrift des Vereines deutscher Ingenieure, 59(11), (1915), 221-224.

3. Bickley, W.: Deflexions and vibrations of a circular elastic plate under tension. Phil. Mag., 59, (1933), 777-797.

4. Fischer, U.: Untersuchung der elastischen Beulung von Kreisringplatten unter der Wirkung rotationssymmetrischer Randkräfte. Ph.D. thesis, TH Magdeburg, 1965.

5. Majumdar, S.: Buckling of a thin annular plate under uniform compression. AIAA Journal, 9, (1971), 1701-1707.

6. Pardoen, G.: Vibration and buckling analysis of axisymmetric polar orthotropic circular plates. Computers \& Structures, 4, (1974), 951-960.

7. Ramaiah, G. and Vijayakumar, K.: Elastic stability of annular plates under uniform compressive forces along the outer edge. AIAA Journal, 13, (1975), 832-834.

8. Chen, L.-W. and Dong, J.-L.: Vibrations of an initially stressed transversely isotropic circular thick plate. International Journal of Mechanical Sciences, 26(4), (1984), 253-263.

9. Wang, C. M. and Aung, T. M.: Buckling of circular plates under intermediate and edge radial loads. Thin-Walled Structures, 43, (2005), 1926-1933.

10. Zhang, Y., Chen, L., Swaddiwudhipong, S., Liu, Z., ET Al.: Buckling Deformation of Annular Plates Describing Natural Forms. International Journal of Structural Stability and Dynamics, 14(1), (2014), 1350054[15 pages].

11. Jillella, N. and Peddieson, J.: Elastic Stability of Annular Thin Plates with One Free Edge. Journal of Structures, 2013.

12. Thevendran, V. and Wang, C. M.: Buckling of Annular Plates Elastically Restrained against Rotation along Edges. Thin-Walled Structures, 25(3), (1996), 231-246.

13. Laura, P. A. A., Gutiérrez, R. H., Sanzi, H. C., and Elvira, G.: Buckling of circular, solid and annular plates with an intermediate circular support. Ocean Engineering, 27, (2000), 749-755.

14. WANG, C. Y. and WANG, C. M.: Buckling of circular plates with an internal ring support and elastically restrained edges. Thin-Walled Structures, 39, (2001), 821-825.

15. Reddy, J. N., WAng, C. M., and Wang, C. Y.: Exact Solutions for Buckling of Structural Members. CRC Press, 2005.

16. Wang, C. M. and Aung, T. M.: Buckling of Circular Mindlin Plates with an Internal Ring Support and Elastically Restrained Edge. Journal of Engineering Mechanics, 131(4), (2005), 359-366.

17. RAO, L. B. and RAO, C. K.: Buckling of Circular Plates with an Internal Elastic Ring Support and Elastically Restrained Guided Edge Against Translation. Mechanics Based Design of Structures and Machines: An International Journal, 37(1), (2009), 60-72.

18. RAO, L. B. and RAO, C. K.: Buckling Analysis of Circular Plates with Elastically Restrained Edges and Resting on Internal Elastic Ring Support. Mechanics Based Design of Structures and Machines: An International Journal, 38(4), (2010), 440-452.

19. RAO, L. B. and RAO, C. K.: Buckling of circular plates with an internal elastic ring support and outer edge restrained against translation. Journal of Engineering Science and Technology, 7(3), (2012), 393-401. 
20. RAO, L. B. and RAO, C. K.: Fundamental buckling of circular plates with elastically restrained edges and resting on concentric rigid ring support. Frontiers of Mechanical Engineering, 8(3), (2013), 291-297.

21. RAO, L. B. and RAO, C. K.: Buckling of circular plate with foundation and elastic edge. International Journal of Mechanics and Materials in Design.

22. Troitsky, M.: Stiffened Plates. Elsevier, 1971.

23. Simitses, G. J. and Blackmon, C. M.: Buckling of eccentrically stiffened, thin circular plates. AIAA Journal, 7(6), (1969), 1200-1202.

24. Srinivasan, R. S. and Thiruvenkatachari, V.: Static and Dynamic Analysis of Stiffened Plates. Computers 83 Structures, 21(3), (1985), 395-403.

25. Phillips, J. S. and Carney, J. F.: Stability of an Annular Plate Reinforced With a Surrounding Edge Beam. Journal of Applied Mechanics, ASME, 41(2), (1974), 497-501.

26. Turvey, G. J. and Der Avanessien, N. G. V.: Axisymmetric elasto-plastic large deflection response of ring siffened circular plates. International Journal of Mechanical Sciences, 31(11-12), (1989), 905-924.

27. Turvey, G. J. and Salehi, M.: Elasto-plastic large deflection response of pressure loaded circular plates stiffened by a single diameter stiffener. Thin-Walled Structures, 46, (2008), 996-1002.

28. Golmakani, M. E. and Mehrabian, M.: Nonlinear bending analysis of ring-stiffened circular and annular general angle-ply laminated plates with various boundary conditions. Mechanics Research Communications, 59, (2014), 42-50.

29. Rossettos, J. N. and Miller, W. H.: On the Buckling of Ring-Stiffened Circular Plates. Journal of Applied Mechanics, ASME, 51(3), (1984), 689-691.

30. Rossettos, J. N. and YAng, G.: Asymmetric Buckling of Ring Stiffened Circular Plates. Journal of Applied Mechanics, ASME, 53(2), (1986), 475-476.

31. Frostig, Y. and Simitses, G. J.: Effect of boundary conditions and rigidities on the buckling of annular plates. Thin-Walled Structures, 5(4), (1987), 229-246.

32. Frostig, Y. and Simitses, G. J.: Buckling of ring-stiffened multi-annular plates. Computers \& Structures, 29(3), (1988), 519-526.

33. Szilassy, I.: Stability of circular plates with a hole loded on their outer boundary. Ph.D. thesis, University of Miskolc, 1971. (in Hungarian).

34. SzILASSY, I.: Stability of an annular disc loaded on its external flange by an arbitrary force system. Publ. Techn. Univ. Heavy Industry. Ser. D. Natural Sciences, 33, (1976), $31-55$.

35. Vlasov, V. Z.: General theory of shells and its applications in engineering, in: Selected Papers [in Russian]. Vol. 1, Izd. Akad. Nauk SSSR, Moscow, 1962.

36. JEzsó, K.: Cylyndrical shells subjected to non-axisymmetrical loading - a solution procedure. Ph.D. thesis, University of Miskolc, 1980. (in Hungarian).

37. Burmeister, D.: Stability of shell-stiffened and axisymmetrically loaded annular plates. Technische Mechanik, 33(1), (2013), 1-18. 